\title{
The neurobiology of smartphone addiction in emerging adults evaluated using brain morphometry and resting-state functional MRI
}

Aida Abdul Rashid ${ }^{1}$, Subapriya Suppiah ${ }^{1 *}$, Nisha Syed Nasser ${ }^{1,2}$, Hamed Sharifat ${ }^{1}$, Mazlyfarina Mohamad ${ }^{3}$, Jia Ling Loh ${ }^{1}$, Buhari Ibrahim ${ }^{1,4}$, Nur Shahidatul Nabila Ibrahim ${ }^{1}$, Nur Hafizah Mohad Azmi ${ }^{1}$, Ezamin Abdul Rahim ${ }^{1}$, Laila Mastura Ahmad Appandi ${ }^{1}$, Suzana Ab Hamid ${ }^{1}$, Yap Ngee Thai ${ }^{5}$, Siew Mooi Ching ${ }^{6}$ and Fan Kee Hoo ${ }^{7}$

${ }^{1}$ Department of Radiology, Faculty of Medicine and Health Sciences, Universiti Putra Malaysia, Selangor, Malaysia.

${ }^{2}$ Centre for Advanced Research in Imaging, Neuroscience and Genomics, Mahajan Imaging, New Delhi, India.

${ }^{3}$ Centre for Diagnostic and Applied Health Sciences, Faculty of Health Sciences, Universiti Kebangsaan Malaysia, Kuala Lumpur, Malaysia.

${ }^{4}$ Department of Physiology, Faculty of Basic Medical Sciences, Bauchi State University Gadau, Bauchi State, Nigeria.

${ }^{5}$ Department of English Language, Faculty of Modern Language and Communications, Universiti Putra Malaysia, Selangor, Malaysia.

${ }^{6}$ Department of Family Medicine, Faculty of Medicine and Health Sciences, Universiti Putra Malaysia, Selangor, Malaysia.

${ }^{7}$ Neurology Unit, Department of Medicine, Faculty of Medicine and Health Sciences, Universiti Putra Malaysia, Selangor, Malaysia.

* Correspondence: subapriya@upm.edu.my; Tel.: +603 97692512

Received: 31 July 2021; Accepted: 8 October 2021; Published: 27 December 2021

Edited by: Kamalanathan Palaniandy (National University of Malaysia, Malaysia)

Reviewed by: Hamzaini Abdul Hamid (National University of Malaysia, Malaysia); Rafidah Zainon (Universiti Sains Malaysia, Malaysia); Aini Ismafairus Abd Hamid (Universiti Sains Malaysia, Malaysia)

https://doi.org/10.31117/neuroscirn.v4i4.107

Abstract: The characteristics of smartphone addiction (SPA) can be evaluated by neuroimaging studies. Information on the brain structural alterations, and effects on psychosocial wellbeing, however, have not been concurrently evaluated. The aim of this study was to identify abnormalities in gray matter volume using voxel-based morphometry (VBM) and neuronal functional alterations using resting-state functional $\mathrm{MRI}$ ( $\mathrm{rs}-\mathrm{fMRI}$ ) in emerging adults with SPA. We correlated the neuroimaging parameters with indices for psychosocial wellbeing such as depression, anxiety, stress, and impulsivity. Forty participants (20 SPA and 20 age-matched healthy controls) were assessed using VBM and rs-fMRI. The smartphone addiction scale - Malay version (SAS-M) questionnaire scores were used to categorize the SPA and healthy control groups. DASS-21 and BIS-11 questionnaires were used to assess for psychosocial wellbeing and impulsivity, respectively. VBM identified the SPA group to have reduced gray matter volume in the insula and precentral gyrus; and increased grey matter volume in the precuneus relative to controls. A moderate correlation was observed between the precuneus volume and the SAS-M scores. Individuals with SPA showed significant rs-fMRI activations in the precuneus, and posterior cingulate cortex (FWE uncorrected, $p<0.001$ ). The severity of SPA was correlated with depression. Anxiety score was moderately correlated with reduced GMV at the precentral gyrus. Collectively, these results can be used to postulate that the structural and neuronal functional changes in the insula are linked to the neurobiology of SPA that shares similarities with other behavioural addictions. 
Keywords: behavioural addiction; Internet addiction; problematic smartphone use; resting-state functional magnetic resonance imaging; voxel-based morphometry;

C2021 by the Abdul Rashid et al. for use and distribution according to the Creative Commons Attribution (CC BYNC 4.0) license (https://creativecommons.org/licenses/by-nc/4.0/), which permits unrestricted non-commercial use, distribution, and reproduction in any medium, provided the original author and source are credited.

\subsection{INTRODUCTION}

Smartphone addiction (SPA) can be defined as a nonsubstance related technological addiction that involves human-machine interaction and elucidates the tolerance rate, functional impairment, compulsion, and withdrawal symptoms concerning mobile phones (Lin et al., 2014). As a consequence of a rapidly expanding worldwide information technology industry, there has been quick access to the internet and fast distribution of smartphones, leading to maladaptive use resulting in a serious addiction behaviour, distinctly noticeable in a vulnerable group of people, particularly adolescents and emerging adults (Kwon et al., 2013a). SPA is believed to share parallels to other types of addictions, particularly internet addiction disorder (IAD) and substance use disorders (SUD), whereby there are postulated to be converging neurobiological pathways that explain these behavioural addictions (Horvath et al., 2020; Nasser et al., 2020a). In fact, SPA and IAD share many similarities and can almost be considered synonymous with each other. To date, validated questionnaires are commonly used to assess SPA, e.g. the Smartphone Addiction Scale (SAS) that was developed in South Korea (Kwon et al., 2013b) Smartphone Addiction Inventory (SPAI) which was developed in Taiwan (Lin et al., 2014), Smartphone Addiction Scale - Malay version (SAS-M) that was developed in Malaysia (Ching et al., 2015), and the Smartphone Addiction Scale - Short Version (SAS-SV) that has been validated for use among emerging adults in the U.S. (Harris et al., 2020). Despite having these instruments as tools for assessing SPA, there is still debate on whether it is a true type of addiction. Hence, neuroimaging studies have been utilised to investigate the underpinnings of this disorder.

One such neuroimaging tool is functional magnetic resonance imaging (fMRI), which can objectively identify the patterns of brain activation that occur during rest or when performing an in-scanner task. This technology enables a non-invasive examination of the in vivo brain function - by measuring regional blood oxygen gradient that acts as a surrogate for evaluating neuronal function (Nasser et al., 2020a). The blood oxygen level-dependent (BOLD) imaging technique used in $\mathrm{fMRI}$ produces distinct signals in the brain based on activations of certain brain regions, i.e., regions of increased cerebral oxygen levels (Nasser et al., 2020a; Zhu et al., 2015). The signals are weaker in the resting-state $\mathrm{fMRI}$ ( $\mathrm{rs}$-fMRI) than during a task-based fMRI scan (Liu et al., 2010). Nonetheless, image processing software can gauge alterations in regional brain activations during rest, particularly in brain nodes involving the default mode network (DMN), salience network or dorsal attention network (DAN), and the limbic network. Based on a priori knowledge from previous fMRI studies, it has been noted that certain neural regions are robustly associated with addictive behaviours, namely the prefrontal cortices (PFC), the insula, the anterior cingulate cortex (ACC), inferior parietal lobe (iPL) and inferior temporal lobes, the precuneus, thalamus and lingual gyrus, and cerebellum (Ding et al., 2013; Horvath et al., 2020; Nasser et al., 2020a)

Additionally, morphological changes of the brain can be non-invasively assessed using an MRI analysis technique called voxel-based morphometry (VBM). VBM is a computational method for assessing anatomical sections of the neuronal cortices and measuring differences in local concentrations of brain tissue, particularly the grey matter (Connolly et al., 2013; Ko et al., 2015). Voxel-wise comparison of multiple brain images such as grey matter density (GMD) and grey matter volume (GMV) - that are inversely related to one another - have been correlated with addiction ( $\mathrm{He}$ et al., 2017). In individuals with SPA and IAD, decreased GMV has been detected in the left anterior insula, inferior temporal lobe, and parahippocampal cortex (Horvath et al., 2020), orbitofrontal cortex (OFC)( $\mathrm{Du}, 2016)$, right superior frontal gyrus (sFG), right inferior frontal gyrus (iFG), and bilateral thalamus (Wang et al., 2016). Alternatively, increased GMV was found in bilateral putamen and right nucleus accumbens ( $\mathrm{Du}, 2016)$. Furthermore, adolescents with IAD demonstrated decreased GMD in the left ACC, left PCC, left insula, left lingual gyrus (Zhu et al., 2015), and bilateral amygdala (Ko et al., 2015; Weinstein, 2017).

Apart from functional and structural brain changes, many addictions have been correlated with psychosocial wellbeing as they can adversely affect adolescents and 
emerging adults. Depression and anxiety have been identified as independent positive predictors of SPA (Boumosleh \& Jaalouk, 2017). Additionally, many addiction disorders have been implicated as dysfunctional behaviour caused by a lack of impulse control. As one of the psychosocial functioning indexes, impulsivity has been implicated as an essential characteristic of internet gaming disorder (IGD), which is a specific type of IAD (Ko et al., 2015) as well as in SPA (Kim et al., 2014). Interestingly, impaired rs-fMRI functional connectivity has been detected between areas of the insula and amygdala with the dorsolateral prefrontal cortex (DLPFC) and OFC, consistent with brain regions related to impulsivity (Sharifat et al., 2018).

We hypothesise that there will be altered GMD and GMV of certain regions of the brain similar to our a priori knowledge. We also hypothesise that there will be impaired activations in the insula among individuals with SPA. Additionally, we hypothesise that the severity of SPA can be correlated with certain GMV, GMD, and rsfMRI neuronal activation parameters, as well as psychosocial indices.

The main objective of this study was to identify whether the neurobiology of SPA shared similarities with other types of addictions. We determined to compare the differences in regional GMV and neuronal function in SPA and healthy controls by using VBM and rs-fMRI, respectively. We also determined to correlate the SAS$M$ scores with psychosocial indices to identify whether they correlate with the severity of SPA. We also evaluated the correlation between the psychosocial indices with MRI parameters.

\subsection{MATERIALS AND METHOD}

\subsection{Study design and subject recruitment}

This is a cross-sectional, case-control study design that had received ethical clearance from our institutional ethical committee. Data for this study were collected from November 2017 to November 2018 for fifty emerging adults who were undergraduate university students in our institution (aged 18-25 years). The sampling method was convenience sampling based on a previous database from a cross-sectional study on SPA in the same university that was performed by our research group (Nasser et al., 2020b). In accordance with the Declaration of Helsinki principles, participation in this survey was voluntary, and informed consent was sought from the potential subjects prior to recruitment into the study. It was also made clear to the students that their acceptance or rejection of participation in the study would have no consequences on their examination marks. The subjects were not given any incentives, and all data was anonymised.

\subsection{Inclusion and exclusion criteria}

The inclusion criteria for the subjects included actively registered undergraduate students in the university with right-hand dominance that was sourced from the previously mentioned database. The subjects were required to be active smartphone users who were categorised in SPA group and healthy control group based on their SAS-M scores. The exclusion criteria included students over 25 years old, postgraduate students, students who had major psychological problems, as evaluated by a mental health questionnaire. Such as students who were on chronic medications that may affect the central nervous system or had a history of substance abuse, i.e., alcohol/cigarette, and those who were contraindicated for MRI examination. Relative and absolute contraindications for MRI examination included claustrophobia, persons with irremovable metallic implants in their body that were not MRI compatible, and persons with electronic implants, i.e., pacemaker, cochlear or ear implants and metallic tattoos.

\subsection{Sample size calculation}

A minimum of 35 subjects was calculated based on the sample size calculation formula for a case control study design as below and using the prevalence of $47 \%$ of SPA cases in a similar population as reported by Ching et al. (Ching et al., 2015)

$$
n=r+1\left(p^{*}\right)\left(1-p^{*}\right)(Z \beta-Z \alpha / 2) 2 \div(p 1-p 2) 2
$$

(Whereby, $r=$ ratio of control to case, equal ratio means $r$ will be $1, p^{*}=$ average proportion of exposed cases, $Z \beta=$ standard normal variate for $95 \%$ power $\mathrm{Cl}(1.96), \mathrm{Z} \alpha / 2=$ standard variate at $5 \%$ type 1 error, $\mathrm{p} 1=$ proportion in cases, p2= proportion in control)

\subsection{Smartphone addiction diagnostic criteria and psychosocial assessment}

The subjects were administered with several questionnaires, which included the SAS-M, Depression Anxiety Stress Scale (DASS-21), and Barratt Impulsiveness Scale (BIS-11) questionnaires.

\subsubsection{Smartphone addiction scale- Malay version questionnaire (SAS-M)}

The smartphone addiction scale is a modified short version of the smartphone addiction scale developed by Kwon et al. (Kwon et al., 2013a) This questionnaire was validated in the Malay language by Ching et al. (Ching et al., 2015) The self-completed 33-item questionnaire uses 
a 6-point Likert scale from 1 to 6 (1= strongly disagree to $6=$ strongly agree) to determine the level of SPA. The total score on the SAS-M ranges from a minimum of 48 to a maximum of 288 points. The SAS-M questionnaire exhibited good internal consistency, whereby the Cronbach's alpha coefficient $(\alpha)$ for the total scale was 0.94 , and had a sensitivity of $71.43 \%$, a specificity of $71.03 \%$ for identifying at-risk cases using an optimal cutoff score of $>98$ (Ching et al., 2015) Thus, based upon the evaluation of our subjects' responses, subjects with scores of $>98$ were considered as having SPA. Those with scores of $\leq 98$ were considered less likely to be at risk of SPA and were categorised as healthy controls.

\subsubsection{Depression Anxiety Stress Scale (DASS-21)}

A 21-item self-reported DASS-21 questionnaire was used to evaluate the state of emotional response i.e., depression, anxiety and stress, rated on a fourpoint Likert scale (Lovibond \& Lovibond, 1995) The scores ranged from 0 (did not apply to them at all), to 4 (apply on them most of the time). After the evaluation of the DASS-21 questionnaire, subjects who scored $<9$ for depression, $<7$ for anxiety, $<14$ for stress were considered normal, and those who scored $>20$ for depression, > 14 for anxiety, >25 for stress were considered having a severe psychiatric problem and not recruited for this study.

\subsubsection{Barratt impulsiveness scale (BIS-11)}

The Barratt impulsiveness scale (BIS-11) is a 30 -item selfreported questionnaire to measure impulsiveness on a 4point scale, i.e., a score of $1=$ never/rarely to score $4=a l m o s t$ always/always. The questions were grouped under six first-order factors, namely attention, motor, self-control, cognitive complexity, perseverance, and cognitive instability impulsiveness, and three second-order factors, namely attentional, motor, and non-planning impulsiveness (Patton et al., 1995). We used a cut-off score $\geq 72$ on the BIS-11 (internally consistency, $\alpha=0.83$ ) as a reference standard to measure the prevalence of high impulsivity among our sample population as suggested by Stanford et al. (Stanford et al., 2009).

\subsection{Structural MRI and Voxel-Based Morphometry (VBM) Analysis}

MRI examination was conducted on a Siemens 3.0T scanner (PRISMA, Siemens, Erlangen, Germany). Structural MRI was performed using a 12-channel head coil. High-resolution T1 MPRAGE MRI data were obtained. The parameters of the sequence were as follows: high-resolution T1-weighted magnetizationprepared rapid gradient echo pulse sequence, TR=
$2300 \mathrm{~ms}, \mathrm{TE}=2.27 \mathrm{~ms}, \mathrm{Tl}=1100 \mathrm{~ms}$, number of slices $=160$, ascending sagittal oriented, $F O V=256 \times 256 \mathrm{~mm}^{2}$, matrix size $=256 \times 256$, and slice thickness $=1 \mathrm{~mm}$. Structural images were pre-processed using the VBM toolbox in the Statistical Parametric Mapping software (SPM 12, http://www.fil.ion.ucl.ac.uk/spm/software/spm12).

The T1-weighted images were spatially registered to the Montreal Neurological Institute (MNI) template and the volume for a specific region of interest (ROI) based on $a$ priori knowledge, which included GMD of the limbic region, frontal lobe, insula, PFC, and iPL lobe, as well as the total intracranial volume (TIV), were generated. Bias correction was performed to remove non-uniformities of the image signal intensity. Segmented images of the GMD and GMV were preserved to assess the amount of volume changes based on spatial registration and the modulated images of the grey matter that reflected the tissue volumes. The TIV of each subject was calculated and used as covariates for analysis. Finally, the normalised brain images were smoothed using a Gaussian filter (8mm FWHM). Multiple comparison correction was applied using family-wise error (FWE), thresholding at $p<0.05$. To detect regions that had very low signal, the threshold was dropped to $p<0.001$ in the SPM analysis, which was considered uncorrected for FWE. The software that we utilised calculated the GMD only. Hence, we utilised the formula of grey matter mass (GMM), a constant that equals the GMD x GMV, to detect the GMV as being inversely proportionate to the GMD.

\subsection{Resting-state fMRI and neuronal functional activation analysis}

Functional imaging was performed using an echo-planar imaging (EPI) sequence. The phase encoding direction was from anterior to posterior, with a TR: $3000 \mathrm{msec}$, TE: $30 \mathrm{msec}$, slice thickness $3 \mathrm{~mm}$, FOV $220 \times 220 \mathrm{~mm}^{2}$, voxel size: $2.3 \times 2.3 \times 3 \mathrm{~mm}$, number of slices 34 . Subjects were asked to lie down with their eyes closed but not to fall asleep. Basic vital assessments such as heart rate, blood pressure and respiratory rate measurement were done prior to commencing the rs-fMRI examination.

We conducted whole-brain analysis, followed by seedbased analysis (SBA) using ROls that were set based on $a$ priori knowledge. The functional images were preprocessed with SPM12 software by applying the following steps: slice-timing correction, spatial realignment, co-registration to the $\mathrm{T}_{1}$-weighted anatomical image, spatial normalisation to the $\mathrm{MNI}$ space, and smoothing. 
The cross-correlation coefficients between these seedbased voxels and all other voxels were calculated to generate correlation maps. These correlation maps were converted to Z-value maps using Fisher's r-to-Z transformation to improve the normality of the correlation coefficients. Next, group analyses were performed for the correlation maps of each seed region. A voxel-wise two-sample t-test was performed to compare the correlation maps derived from each seed between the SPA and HC groups. The significance level was set at $p<0.05$, FWE corrected.

\subsection{Statistical Analysis}

SPM 12 and Statistical Package for the Social Sciences (SPSS software Version 25.0, SPSS Inc., Chicago, IL, USA) was used for statistical analysis. The correlation analysis was carried out to determine the association between SAS-M, DASS-21, and BIS-11 with the subjects categorised into SPA and HC groups. Paired t-test was used to compare the differences in brain grey matter in SPA versus $\mathrm{HC}$ subjects using ROI-based analysis of VBM data. A two-sample t-test was conducted to identify regions of brain activation in SPA subjects using SBA of rs-fMRI data. Pearson correlation was performed to assess the relationship between SPA parameters and psychosocial parameters, with VBM dependant variables. The significance level was set at $p$ value less than 0.05 .

\subsection{RESULTS}

We had initially recruited 50 subjects for this study. The data from ten subjects were discarded and not utilised in the final analysis due to severe movement artefacts. Thus, there were 40 subjects in the final analysis with 20 subjects in each group, i.e., SPA group and healthy control (HC) group. The descriptive statistics were used to compare SPA and HC group with psychosocial assessment parameters (Table 1). The average BIS-11 scores for the SPA group were in the increased impulsivity range. The DASS- 21 scores revealed a mild to moderate level of depression and anxiety and a normal level of stress among the SPA group. The subjects' specific smartphone use duration is provided in Table 1. Individuals with SPA spent more time with their smartphones compared to the control group.

In the VBM analysis, there were significant regional differences in GMD between the SPA and HC in specific brain regions at a threshold of $p<0.05$ (FWE corrected) as shown in Table 2. The largest difference was seen in the GMD of the right insula and right PCG in the between group comparison of SPA $>\mathrm{HC}$ (Figure 1a). The largest difference in GMD for HC>SPA group was seen in the left iPL (Figure 1b).

In the rs-fMRI analysis, significant whole-brain activations at FWE $p<0.05$ for the SPA and HC groups were seen in the regions given in Table $\mathbf{3}$ and Table 4, respectively. The highest peak activation was seen in the left precuneus, followed by the left PCC and right calcarine region in the SPA group (Table 3). For betweengroup comparison of SPA $>\mathrm{HC}$ groups, there was activation at the left fusiform gyrus, right superior frontal gyrus, right precuneus, right calcarine gyrus, right superior motor area, bilateral cerebellum, left superior parietal lobe (sPL), and left PCC $(p<0.001$, FWE uncorrected) (Figure 2). No regions survived, i.e., no rsfMRI activation was seen for the HC>SPA group comparison.

Table 1: Comparative statistics of the mean value of the variables for SPA and HC groups.

\begin{tabular}{llcc}
\hline & \multicolumn{1}{c}{$\begin{array}{c}\text { SPA } \\
\text { Minimum - Maximum Value } \\
\text { (mean } \pm \text { SD) }\end{array}$} & $\begin{array}{c}\text { Minimum - Maximum Value } \\
\text { (mean } \pm \text { SD) }\end{array}$ \\
\hline SAS-M Score & $98-179(129.45 \pm 25.35)$ & $46-90(69.15 \pm 14.13)$ \\
\hline BIS-11 (Impulsivity) & $52-84(70.15 \pm 7.69)$ & $52-85(67.95 \pm 10.16)$ \\
\hline \multirow{N}{*}{} & Depression & $2-36(12.7 \pm 8.21)$ & $0-22(6.2 \pm 6.42)$ \\
\cline { 2 - 4 } & Anxiety & $4-24(11.3 \pm 4.65)$ & $0-22(9.6 \pm 6.67)$ \\
\hline \multicolumn{2}{l}{ Duration of smartphone Use (years) } & $2-28(13.4 \pm 6.68)$ & $0-24(7.8 \pm 6.93)$ \\
\hline
\end{tabular}

Note: SPA: Smartphone addiction group, HC: healthy control group 
Table 2: Tabulated values of regional difference in voxel density for SPA $>H C$ group and HC $>$ SPA $(p<0.05, F W E$ corrected).

\begin{tabular}{lcccccc}
\hline SPA $>$ HC & Cluster Peak & Voxel & Peak T & Mean T & STD & P Level (FWE) \\
\hline Insula (Right) & $35-98$ & 14 & 5.3867 & 5.3414 & 0.0021 & $<0.05$ \\
\hline Precentral gyrus (Right) & $-38-7-2$ & 1 & 4.6293 & 4.6293 & 0 & $<0.05$ \\
\hline HC $>$ SPA & Cluster Peak & Voxel & Peak T & Mean T & STD & P Level (FWE) \\
\hline Inferior Parietal Lobe (Left) & $-20-5438$ & 45 & 5.8638 & 5.4973 & 0.1545 & 0.05 \\
\hline
\end{tabular}

Note: SPA: Smartphone addiction group, HC: healthy control group

Table 3: Whole-brain rs-fMRI activation results in SPA group.

\begin{tabular}{lcccccc}
\hline $\begin{array}{l}\text { Activation Smartphone } \\
\text { Addiction (SPA) }\end{array}$ & Cluster Peak & Voxel & Peak T & Mean T & STD & P Level (FWE) \\
\hline Precuneus Left & $-16-4420$ & 171 & 11.8212 & 7.8313 & 0.7924 & 0.05 \\
\hline Precuneus Right & $10-5432$ & 2 & 9.0163 & 8.3638 & 0.928 & 0.05 \\
\hline Cingulum Middle Left & $-4-3038$ & 41 & 8.9897 & 7.6772 & 0.5364 & 0.05 \\
\hline Cingulum Middle Right & $14-835$ & 18 & 9.2304 & 7.7115 & 0.5708 & 0.05 \\
\hline Cingulum Posterior Left & $-12-4223$ & 6 & 9.8493 & 8.3994 & 0.9286 & 0.05 \\
\hline Calcarine Left & -2185 & 3 & 8.8661 & 8.0209 & 0.8406 & 0.05 \\
\hline Calcarine Right & $10-7417$ & 15 & 9.8384 & 7.7036 & 0.7705 & 0.05 \\
\hline Inferior Parietal Left & $-40-4853$ & 2 & 8.7667 & 7.8566 & 1.2843 & 0.05 \\
\hline Cerebellar 4 ${ }^{\text {th }}$ \& 5 $5^{\text {th }}$ Right & $14-42-22$ & 25 & 8.9976 & 7.6881 & 0.5753 & 0.05 \\
\hline Temporal Pole Superior Right & $3812-25$ & 11 & 8.7716 & 7.6936 & 0.5284 & 0.05 \\
\hline
\end{tabular}

Note: SPA: Smartphone addiction group, HC: healthy control group

Table 4: Whole-brain rs-fMRI activation results in healthy control (HC) group

\begin{tabular}{lcccccc}
\hline $\begin{array}{l}\text { Activation Healthy Control } \\
\text { Group (HC) }\end{array}$ & Cluster Peak & Voxel & Peak T & Mean T & STD & P Level (FWE) \\
\hline Putamen Right & $22-2617$ & 293 & 13.3211 & 8.43 & 1.1404 & 0.05 \\
\hline Insular Right & $3422-4$ & 11 & 12.9328 & 8.7379 & 1.8499 & 0.05 \\
\hline Vermis 4th \& 5th & $0-56-19$ & 96 & 11.2953 & 8.0073 & 0.8159 & 0.05 \\
\hline Fusiform Left & $-36-10-25$ & 7 & 11.0147 & 8.0606 & 1.0675 & 0.05 \\
\hline Occipital Middle Right & $38-6035$ & 49 & 10.7381 & 7.9995 & 0.787 & 0.05 \\
\hline Supplementary Motor Area Left & $-12-856$ & 2 & 10.6388 & 7.151 & 0.0112 & 0.05 \\
\hline Putamen Left & $-188-7$ & 45 & 10.4017 & 7.9484 & 0.7352 & 0.05 \\
\hline Orbitofrontal Inferior Left & $-2826-10$ & 3 & 9.7502 & 8.3332 & 1.717 & 0.05 \\
\hline Postcentral Right & $44-2244$ & 16 & 9.3854 & 7.8414 & 0.7767 & 0.05 \\
\hline Cuneus Left & $-12-8832$ & 8 & 8.7585 & 7.688 & 0.4298 & 0.05 \\
\hline
\end{tabular}

Note: SPA: Smartphone addiction group, $\mathrm{HC}$ : healthy control group

Table 5. Correlation of psychosocial indices and duration of smartphone use with SAS-M scores.

\begin{tabular}{|c|c|c|c|c|c|c|c|c|c|c|}
\hline \multirow{2}{*}{$n=40$} & \multicolumn{2}{|c|}{ BIS Score } & \multicolumn{2}{|c|}{ Depression Score } & \multicolumn{2}{|c|}{ Anxiety Score } & \multicolumn{2}{|c|}{ Stress Score } & \multicolumn{2}{|c|}{ Duration of Use } \\
\hline & $r$ Value & $P$ Value & rValue & $P$ Value & $r$ Value & $P$ Value & $r$ Value & $P$ Value & $r$ Value & $P$ Value \\
\hline SAS-M Score & 0.219 & 0.174 & $0.431 * *$ & 0.006 & 0.200 & 0.217 & 0.285 & 0.075 & 0.274 & 0.087 \\
\hline
\end{tabular}

** Correlation is significant at 0.05 level (2-tailed) 
Table 6. Correlation of SPA and neuropsychological parameters with VBM dependant variables.

\begin{tabular}{|c|c|c|c|c|c|c|c|c|c|c|c|c|}
\hline \multirow{2}{*}{$n=40$} & \multicolumn{2}{|c|}{$\begin{array}{c}\text { BIS } \\
\text { Score }\end{array}$} & \multicolumn{2}{|c|}{$\begin{array}{l}\text { Depression } \\
\text { Score }\end{array}$} & \multicolumn{2}{|c|}{$\begin{array}{c}\text { Anxiety } \\
\text { Score }\end{array}$} & \multicolumn{2}{|c|}{$\begin{array}{l}\text { Stress } \\
\text { Score }\end{array}$} & \multicolumn{2}{|c|}{$\begin{array}{c}\text { SAS-M } \\
\text { Score }\end{array}$} & \multicolumn{2}{|c|}{$\begin{array}{c}\text { Duration } \\
\text { of Use }\end{array}$} \\
\hline & $r$ Value & $\begin{array}{c}P \\
\text { Value }\end{array}$ & $\begin{array}{c}r \\
\text { Value }\end{array}$ & $\begin{array}{c}P \\
\text { Value }\end{array}$ & $r$ Value & $\begin{array}{c}P \\
\text { Value }\end{array}$ & $r$ Value & $\begin{array}{c}P \\
\text { Value }\end{array}$ & $r$ Value & $\begin{array}{c}P \\
\text { Value }\end{array}$ & $\begin{array}{c}r \\
\text { Value }\end{array}$ & $P$ Value \\
\hline GMV & -0.042 & 0.798 & -0.138 & 0.395 & -0.014 & 0.93 & -0.109 & 0.505 & -0.087 & 0.592 & 0.014 & 0.934 \\
\hline TIV & -0.096 & 0.556 & -0.144 & 0.376 & 0.075 & 0.646 & 0.01 & 0.951 & -0.111 & 0.497 & -0.064 & 0.696 \\
\hline BPV & -0.013 & 0.936 & -0.049 & 0.762 & 0.033 & 0.84 & 0.015 & 0.927 & -0.035 & 0.831 & 0.035 & 0.829 \\
\hline Amyg & -0.031 & 0.85 & -0.149 & 0.357 & -0.042 & 0.797 & -0.121 & 0.455 & -0.105 & 0.518 & -0.05 & 0.759 \\
\hline Precuneus & -0.015 & 0.926 & -0.216 & 0.18 & -0.101 & 0.534 & -0.183 & 0.258 & $-0.284 * *$ & 0.076 & -0.025 & 0.881 \\
\hline PFC & -0.109 & 0.503 & -0.087 & 0.594 & 0.029 & 0.86 & -0.093 & 0.568 & -0.093 & 0.567 & -0.123 & 0.451 \\
\hline Insula (R) & -0.082 & 0.617 & -0.038 & 0.816 & 0.121 & 0.458 & -0.132 & 0.415 & -0.062 & 0.705 & -0.06 & 0.713 \\
\hline PCG (R) & 0.09 & 0.582 & -0.027 & 0.87 & $0.283^{* *}$ & 0.077 & 0.27 & 0.092 & -0.092 & 0.572 & 0.055 & 0.738 \\
\hline Parietal (L) & -0.093 & 0.568 & -0.23 & 0.154 & -0.102 & 0.53 & -0.213 & 0.188 & -0.211 & 0.192 & 0.123 & 0.449 \\
\hline
\end{tabular}

Note Value with*Correlation is significant at the 0.01 level (2 -tailed), ${ }^{* *}$ Correlation is significant at 0.05 level (2-tailed). Gray matter volume "GMV", total intracranial volume "TIV", brain parenchymal volume "BPV", amygdala volume "Amyg", precuneus volume "Precuneus", prefrontal cortex volume "PFC", right insula volume "Insula (R)", right precentral gyrus volume "PCG (R)", and left parietal volume "Parietal (L)".

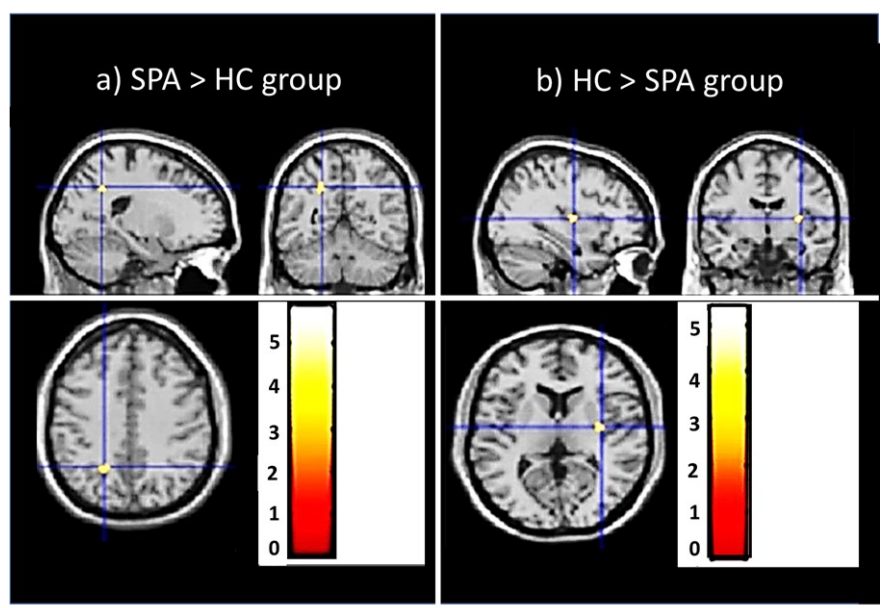

Figure 1: Voxel-based morphometry showing greater GMD in the (a) right insula in SPA $>\mathrm{HC}$ comparison and the (b) left inferior parietal lobe in HC>SPA comparison (significance set at $\mathrm{p}<0.05$, FWE corrected).

Pearson's correlation test revealed that the SAS-M score was negatively correlated with the GMD of the precuneus (Pearson's product-moment correlation coefficient, $r=-0.284, p=0.076)$, which, although was not statistically significant, indicated a moderate positive correlation as illustrated in the scatter plot in Figure $\mathbf{3 .}$ Depression was significantly correlated with the severity of SPA, i.e., the SAS-M scores, as shown in Table 5. At the same time, the anxiety score was positively correlated with the GMD of the right PCG (Pearson's productmoment correlation coefficient, $r=0.283, p=0.077)$, which, although was not statistically significant, indicated a moderate positive correlation (Table 6).

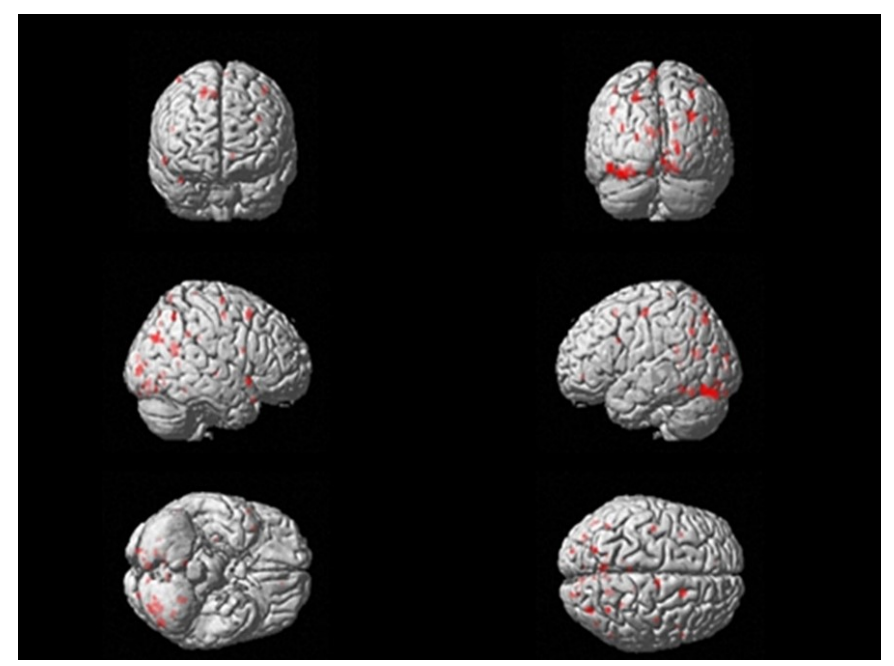

Figure 2: Regions of brain activation SPA $>H C(p<0.001, F W E$ uncorrected).

\subsection{DISCUSSION}

Our multimodal MRI study, using morphological data based on structural MRI images and functional data from rs-fMRI, was able to evaluate the intrinsic neural activity in subjects with SPA. We detected that individuals with SPA had decreased or lower GMV at the right insula; and increased or higher GMV, as seen by the lower GMD, in the precuneus. Horvath et al. also identified reduced GMV of the insula, but on the left side, and associated this with impaired salience attribution and attentional resources as well as increased cravings among individuals with SPA (Horvath et al., 2020). Decreased GMV of the insula has also been 


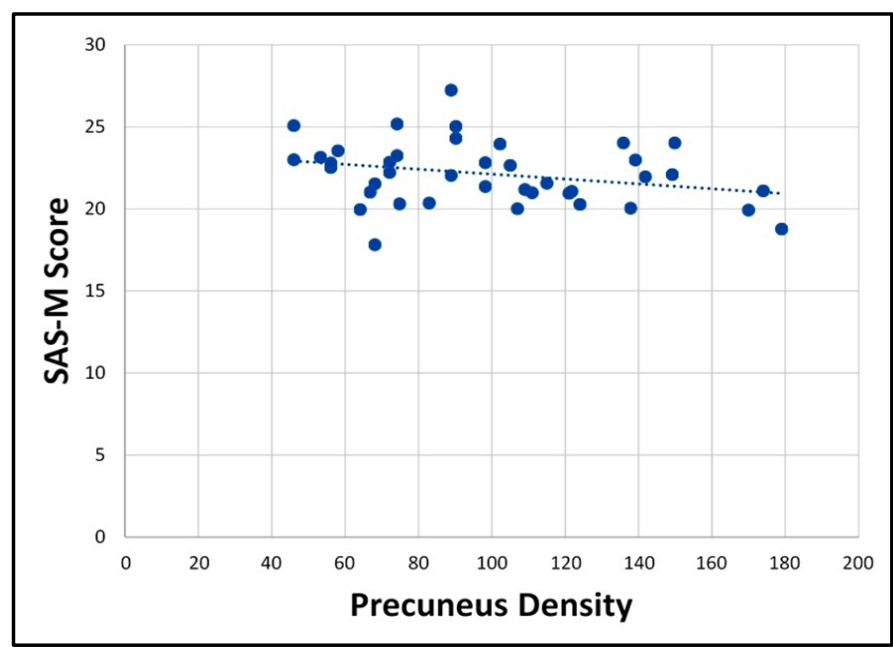

Figure 3. Simple scatter plot showing the correlation of the grey matter density of the Precuneus with the SAS-M scores.

reported in substance-related addictions (Suckling \& Nestor, 2017). Hence, this explains why the insula, which is part of the DAN or salience network, is more activated in the individuals with SPA than the healthy controls. GMV reductions of the insula in addiction studies have been hypothesised to be due to maladaptive social behaviours and increased impulsivity (Noël et al., 2013). In addition, the insula is known to heavily project to the amygdala, which is a known hub implicated in impulsiveness among subjects with IGD (므, 2016).

Conversely, higher GMV of the precuneus was noted among individuals with SPA. Furthermore, we observed that the severity of SPA was negatively correlated with the GMD of the precuneus, i.e., positively correlated with the GMV of the precuneus, although this was not proven to be statistically significant. Our finding shares some similarities with a study by Dong et al., which had detected that IGD severity was positively correlated with the GMV of the precuneus (Dong et al., 2020). It was postulated that this abnormality of the precuneus might be due to the derangement of the role of the precuneus, which in normal conditions acts as a platform for integrating potential contradictory information between executive control and sub-cortical cravings (Dong et al., 2020).

Functionally, there was a deactivation of the insula among individuals with SPA. However, several regions of the DMN were more activated in individuals with SPA than the healthy controls, i.e., the right precuneus and the left PCC. The insula is part of the cerebral cortex, located adjacent to the basal ganglia and the Sylvian fissure, and in many studies has demonstrated an integral role in salience network; related to positive desire associated with addiction. According to Volkow et al., the insula and ACC have been described as essential nodes of the salience network that mediate the DMN (Volkow et al., 2016). Similarly, a meta-analysis of VBM studies has revealed significant decreases of the GMV in individuals having SUD, namely in the insula, ventromedial PFC, $\mathrm{iFG}$, pregenual $\mathrm{ACC}$, and anterior thalamus (Ersche et al., 2013).

The SAS-M scores were significant correlation with depression. Thus, this may indicate that individuals with increasing severity of SPA have an association with depression. A cross-sectional study of 388 undergraduate university students evaluated using the SPAI questionnaire demonstrated that depression and anxiety were significant predictors of SPA (Boumosleh \& Jaalouk, 2017). However, in this study, we cannot postulate a causality effect because of the limitation of our study design.

The clinical implication of our findings is that we were able to demonstrate brain regions to have structural changes and functional abnormalities that were similar to other types of behavioural addictions. We also propose that increased GMV of the precuneus can act as an imaging biomarker of SPA, because it can occur with increasing severity of SPA. As studied in other neuropsychiatric conditions such as Alzheimer's disease $(A D), r s-f M R I$ analysis of regions of the DMN is used to potentially diagnose the early stage of the disorder, thus predicting the transition from the prodromal phase to full-blown disease (Ibrahim et al., 2021). Similarly, future directions are proposed to recognise smartphone and internet addictions at an early stage in an objective manner. Furthermore, psycho-behavioural intervention is best assessed using these neuroimaging studies and potentially reduces the high costs of drug intervention programmes. Overall, our results can highlight this type of addiction's seriousness to the administrators of higher learning institutions and impact legislations.

The limitation of this study is the small sampling size, which was underpowered by the limited number of subjects recruited. We recommend that future studies recruit more subjects using our scan protocol to evaluate SPA further. Also, using a convenience sampling method does not enable us to evaluate individuals with similar severity and stage of addiction, which potentially creates heterogeneity of our data and inhibits us from achieving truly significant correlation results. The cut-off scores used for the Malay version of the SAS questionnaire is also another limitation for categorising our participants and future improvements in the accuracy of the 
questionnaire may be helpful. Furthermore, the indicators for SPA were assessed via self-report data rather than objectively recorded data. This can be rectified by performing analysis based on data from the subjects' smartphone applications and clinical interviews with friends or family who can impartially evaluate the subjects.

\subsection{CONCLUSIONS}

We were able to identify that the neurobiology of SPA shared similarities with other types of addictions, as evidenced by reduced grey matter volume in the insula and precentral gyrus. This indicated that they had reduced executive control, which caused impulsivity to continue their addictive behaviour similar to substance abuse addiction. The severity of addiction based on SAS$M$ scores were correlated with precuneus volume and activations, which implied increased salience to addictive behaviour among the smartphone addicts. The severity of SPA was correlated with depression, which needs longitudinal study to determine causality. This study gives evidence-based proof that smartphone addiction has similarities to other types of behavioural addiction and substance use disorders.

Ethical clearance: This study was approved by the Ethics Committee of Research Involving Human Subjects of Universiti Putra Malaysia (JKEUPM UPM/TNCPI/RMC/JKEUPM/ 1.4.18.2).

Acknowledgement: The grants that funded this work were from the Universiti Putra Malaysia research grant i.e., Geran
Putra GP/2017/9549800 and GP-IPS/2017/9580800. The research was also partly funded by the Fundamental Research Grant Scheme (FRGS 06-02-14-1497FR/5524581) with grant number 5540244 that was awarded by the Ministry of Higher Education, Malaysia. The authors would like to thank Associate Professor Dr. Ahmad Nazlim Yusoff from the Faculty of Health Sciences, Universiti Kebangsaan Malaysia, Malaysia, for his expert consultation on data analysis. We also acknowledge Puan Salasiah Mustafa from the Centre of Diagnostic Nuclear Imaging, Universiti Putra Malaysia, Malaysia, who facilitated our data collection process. We would like to extend our gratitude to the students and lecturers from the various faculties in UPM who directly or indirectly assisted in contributing to the data collection.

Author Contributions: AAR, NSN, HS, and LJL were involved in data collection and data analysis. AAR also performed data interpretation and prepared the first draft of the manuscript. SS was responsible for the conceptual framework and study design, secured financial support, conducted data interpretation and supervised the project. $\mathrm{MM}$ also helped in formulating the conceptual framework and study design and the data analysis and interpretation. BI, NSNI, and NHMA conducted the literature search, data analysis, and data interpretation. EAR, LMAA, and SAH were involved in the study design, project supervision, and verification of the analytical methods. YNT was involved in securing part of the financial support for this study and data collection. CSM and HFK were involved in the conceptual framework, verification of analytical methods, and data interpretation. All the authors were involved in editing and verifying the final completed manuscript.

Conflict of Interest: The authors declare that there is no conflict of interest regarding the publication of this work.

\section{References}

Ching, S. M., Yee, A., Ramachandran, V., Sazlly Lim, S. M., Wan Sulaiman, W. A., Foo, Y. L., \& Hoo, F. K. (2015). Validation of a Malay version of the smartphone addiction scale among medical students in Malaysia. PLOS ONE, 10(10), e0139337. https://doi.org/10.1371/journal.pone.0139337

Connolly, C. G., Bell, R. P., Foxe, J. J., \& Garavan, H. (2013). Dissociated grey matter changes with prolonged addiction and extended abstinence in cocaine users. PLOS ONE, 8(3), e59645. https://doi.org/10.1371/journal.pone.0059645

Ding, W., Sun, J., Sun, Y., Zhou, Y., Li, L., Xu, J., \& Du, Y. (2013). Altered default network resting-state functional connectivity in adolescents with internet gaming addiction. PLOS ONE, 8(3), e59902. https://doi.org/10.1371/journal.pone.0059902

Dong, G., Wang, M., Wang, Z., Zheng, H., Du, X., \& Potenza, M. N. (2020). Addiction severity modulates the precuneus involvement in internet gaming disorder: Functionality, morphology and effective connectivity. Progress in NeuroPsychopharmacology and Biological Psychiatry, 98, 109829. https://doi.org/10.1016/j.pnpbp.2019.109829

Du, X., Qi, X., Yang, Y., Du, G., Gao, P., Zhang, Y., Qin, W., Li, X., \& Zhang, Q. (2016). Altered structural correlates of impulsivity in adolescents with internet gaming disorder. Frontiers in Human Neuroscience, 10. https://doi.org/10.3389/fnhum.2016.00004

Ersche, K. D., Williams, G. B., Robbins, T. W., \& Bullmore, E. T. (2013). Meta-analysis of structural brain abnormalities associated with stimulant drug dependence and neuroimaging of addiction vulnerability and resilience. Current Opinion in Neurobiology, 23(4), 615-624. https://doi.org/10.1016/j.conb.2013.02.017

Harris, B., McCredie, M., \& Fields, S. (2020). Examining the psychometric properties of the smartphone addiction scale and its short version for use with emerging adults in the U.S. Computers in Human Behavior Reports, 1, 100011. https://doi.org/10.1016/i.chbr.2020.100011 
He, Q., Turel, O., \& Bechara, A. (2017). Brain anatomy alterations associated with social networking site (SNS) addiction. Scientific Reports, 7(1). https://doi.org/10.1038/srep45064

Horvath, J., Mundinger, C., Schmitgen, M. M., Wolf, N. D., Sambataro, F., Hirjak, D., Kubera, K. M., Koenig, J., \& Christian Wolf, R. (2020). Structural and functional correlates of smartphone addiction. Addictive Behaviors, 105, 106334. https://doi.org/10.1016/i.addbeh.2020.106334

Ibrahim, B., Nasser, N. S., Ibrahim, N., Mohamed, M., Hassan, H. A., Saripan, M. I., \& Suppiah, S. (2020). Diagnostic power of resting-state $\mathrm{fMRI}$ for detection of network connectivity in Alzheimer's disease and mild cognitive impairment: $A$ systematic review. Human Brain Mapping, 42(9):2941-2968. https://doi.org/10.1002/hbm.25369

Kim, S. M., Huh, H. J., Cho, H., Kwon, M., Choi, J. H., Ahn, H. J., Lee, S. W., Kim, Y. J., \& Kim, D. J. (2014). The effect of depression, impulsivity, and resilience on smartphone addiction in university students. Journal of Korean Neuropsychiatric Association, 53(4), 214. https://doi.org/10.4306/jknpa.2014.53.4.214

Ko, C., Hsieh, T., Wang, P., Lin, W., Yen, C., Chen, C., \& Yen, J. (2015). Altered gray matter density and disrupted functional connectivity of the amygdala in adults with internet gaming disorder. Progress in Neuro-Psychopharmacology and Biological Psychiatry, 57, 185-192. https://doi.org/10.1016/j.pnpbp.2014.11.003

Kwon, M., Kim, D., Cho, H., \& Yang, S. (2013a). The smartphone addiction scale: Development and validation of a short version for adolescents. PLOS ONE, 8(12), e83558. https://doi.org/10.1371/journal.pone.0083558

Kwon, M., Lee, J., Won, W., Park, J., Min, J., Hahn, C., Gu, X., Choi, J., \& Kim, D. (2013b). Development and validation of a smartphone addiction scale (SAS). PLOS ONE, 8(2), e56936. https://doi.org/10.1371/journal.pone.0056936

Lin, Y., Chang, L., Lee, Y., Tseng, H., Kuo, T. B., \& Chen, S. (2014). Development and validation of the smartphone addiction inventory (SPAI). PLOS ONE, 9(6), e98312. https://doi.org/10.1371/journal.pone.0098312

Liu, B., Song, M., Li, J., Liu, Y., Li, K., Yu, C., \& Jiang, T. (2010). Prefrontal-related functional connectivities within the default network are modulated by COMT val158met in healthy young adults. Journal of Neuroscience, 30(1), 64-69. https://doi.org/10.1523/jneurosci.3941-09.2010

Lovibond, S. H., \& Lovibond, P. F. (1995). Manual for the depression anxiety stress scales (2nd ed.). Sydney: Psychology Foundation.

Boumosleh, J.M., \& Jaalouk, D. (2017). Depression, anxiety, and smartphone addiction in university students- A cross sectional study. PLOS ONE, 12(8), e0182239. https://doi.org/10.1371/journal.pone.0182239

Nasser, N. S., Ling, L. J., Rashid, A. A., Sharifat, H., Ahmad, U., Ibrahim, B., Mustafa, S., Kee, H. F., Mooi, C. S., \& Suppiah, S. (2020a). A cross-sectional survey on smartphone usage pattern, the level of mobile phone dependence and psychosocial effects among undergraduate students in a Malaysian University. The Medical Journal of Malaysia, 75(4), $356-362$. https://doi.org/10.1101/2020.01.06.20016592

Nasser, N. S., Sharifat, H., Rashid, A. A., Hamid, S. A., Rahim, E. A., Loh, J. L., Ching, S. M., Hoo, F. K., Ismail, S., Tyagi, R., Mohamad, M., \& Suppiah, S. (2020b). Cue-reactivity among young adults with problematic Instagram use in response to Instagram-themed risky behavior cues: a pilot fMRI study. Frontiers in Psychology, 11, 556060. https://doi.org/10.3389/fpsyg.2020.556060

Noël, X., Brevers, D., \& Bechara, A. (2013). A triadic neurocognitive approach to addiction for clinical interventions. Frontiers in Psychiatry, 4. https://doi.org/10.3389/fpsyt.2013.00179

Patton, J. H., Stanford, M. S., \& Barratt, E. S. (1995). Barratt impulsiveness scale-11 (BIS-11). PsycTESTS Dataset. https://doi.org/10.1037/t05661-000

Stanford, M. S., Mathias, C. W., Dougherty, D. M., Lake, S. L., Anderson, N. E., \& Patton, J. H. (2009). Fifty years of the Barratt impulsiveness scale: An update and review. Personality and Individual Differences, 47(5), 385-395. https://doi.org/10.1016/j.paid.2009.04.008

Sharifat, H., Rashid, A. A., \& Suppiah, S. (2018). Systematic review of the utility of functional MRI to investigate internet addiction disorder: Recent updates on resting state and task-based fMRI. Malaysian Journal of Medicine and Health Sciences, 14(1), 21-33.

Suckling, J., \& Nestor, L. J. (2016). The neurobiology of addiction: The perspective from magnetic resonance imaging present and future. Addiction, 112(2), 360-369. https://doi.org/10.1111/add.13474

Volkow, N. D., Koob, G. F., \& McLellan, A. T. (2016). Neurobiologic advances from the brain disease model of addiction. New England Journal of Medicine, 374(4), 363-371. https://doi.org/10.1056/nejmra1511480

Wang, Y., Zou, Z., Song, H., Xu, X., Wang, H., D’Oleire Uquillas, F., \& Huang, X. (2016). Altered gray matter volume and white matter integrity in college students with mobile phone dependence. Frontiers in Psychology, 7. https://doi.org/10.3389/fpsyg.2016.00597

Weinstein, A. M. (2017). An update overview on brain imaging studies of internet gaming disorder. Frontiers in Psychiatry, 8. https://doi.org/10.3389/fpsyt.2017.00185

Zhu, Y., Zhang, H., \& Tian, M. (2015). Molecular and functional imaging of internet addiction. BioMed Research International, 2015, 1-9. https://doi.org/10.1155/2015/37867 